\title{
Aktiviertes Vitamin D senkt die Morbidität und Mortalität bei sHPT
}

Viel früher als lange Zeit angenommen, steigt das kardiovaskuläre Risiko im Verlauf einer chronischen Nierenerkrankung. Dabei spielt der sekundäre Hyperparathyreoidismus (sHPT) schon lange vor Beginn der Dialyse eine zentrale Rolle. „Wahrscheinlich ist der Abfall des 1,25-Vitamin-D-Serumspiegels der wesentliche Trigger des frühen Hyperparathyreoidismus“, sagte PD Dr. Markus Ketteler, Coburg. In der Praxis bedeutet das: Schon bei Patienten mit einer glomerulären Filtrationsrate unter $60 \mathrm{ml} / \mathrm{min}$ finden sich häufig erhöhte Parathormonwerte und kritisch erniedrigte 1,25-Vitamin-D-Serumspiegel. Die Phosphatwerte dagegen steigen erst später, in der Regel erst im Stadium 4 der Niereninsuffizienz an (5).

Probleme beginnen schon relativ früh Ein 1,25-Vitamin-D-Mangel ist demnach ein früher Risikofaktor für den sekundären Hyperparathyreoidismus. Dies bestätigten auch die vorläufigen Daten der prospektiven SEEK ${ }^{1}$-Studie $(\mathrm{n}=1814$ ), die erstmals auf dem Kongress der amerikanischen Gesellschaft für Nephrologie
(ASN) im Jahr 2005 präsentiert wurden. Je stärker sich die Nierenfunktion verschlechterte, sich die glomeruläre Filtrationsrate (GFR) also verringerte, desto mehr Patienten wiesen einen gestörten 1,25-Vitamin-D-Stoffwechsel auf. Bei immerhin 30 bis 50 \% der Patienten im Stadium 2 des Nierenversagens mit einer glomerulären Filtrationsrate zwischen 60 und $90 \mathrm{ml} / \mathrm{min}$ ist nach Angaben von SEEK ein 1,25-Vitamin-D-Mangel zu erwarten - nach Einschätzung Kettelers ein vergleichsweise großer Anteil. Selbst wenn die Kalzium- und Phosphatspiegel zu Beginn des sekundären Hyperparathyreoidismus im Stadium 2 oder 3 des Nierenversagens in der Regel noch annähernd normal sind, ist der Mineralstoffwechsel bereits gestört. Dies kann nicht nur zur renalen Osteodystrophie führen, sondern potenziell auch zu einem erhöhten kardiovaskulären Risiko beitragen.

\section{Vitamin-D-Mangel so früh wie möglich ausgleichen \\ „Man sollte daher einen 1,25-Vitamin- D-Mangel so früh wie möglich ausglei-}

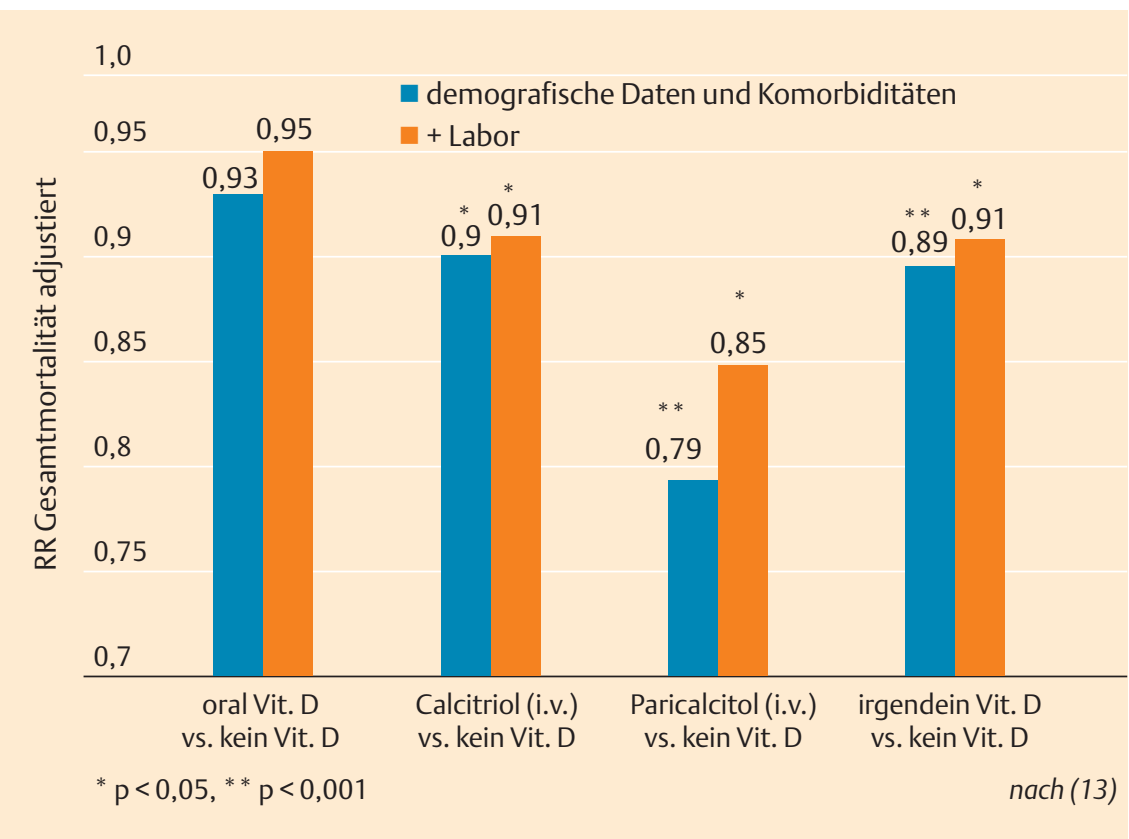

Abb. 1 DOPPS2: Vitamin-D-Therapie und Mortalität (adjustiert hinsichtlich demografischer Daten, Komorbiditäten und Laborwerte) chen" riet Ketteler. Ziel dabei ist zunächst, die Parathormonwerte zu normalisieren, um knochenpathologische und möglicherweise auch kardiovaskuläre Auswirkungen des gestörten Kalzium- und Phosphat-Haushalts auszugleichen. Um normale PTH-Serumwerte aufrecht $\mathrm{zu}$ erhalten, erscheinen $1,25(\mathrm{OH})_{2}$-Vitamin-D-Spiegel von 35-40 $\mathrm{pg} / \mathrm{ml}$ erforderlich zu sein, fasste Ketteler die Ergebnisse von SEEK zusammen. Um dies zu erreichen, reicht jedoch in der Regel die alleinige Gabe von Cholecalciferol bzw. 25(OH)-Vitamin-D nicht aus. Denn bei abnehmender Nierenfunktion kann dieses nicht mehr in ausreichenden Mengen hydroxyliert und damit aktiviert werden. Daher ist bei diesen Patienten in der Regel eine Therapie mit einem aktivierten Vitamin-DAnalogon indiziert.

\section{„To D or not to D“ ist eigentlich keine} Frage

Auch für Prof. Jan Galle, Lüdenscheid, stellt sich die Frage um den Nutzen einer Vitamin-D-Supplementation nicht wirklich. Inzwischen sprechen zu viele experimentelle und klinische Daten für die pleiotropen protektiven Effekte einer Aktivierung der Vitamin-D-Rezeptoren auf Knochen, Nieren, Darm, Pankreas, Immunsystem, natürlich die Parathyroidea und vor allem auf das kardiovaskuläre System.

So moduliert aktives Vitamin D beispielsweise das Renin-Angiotensin-System (RAS) (12) und damit den Blutdruck. Darüber hinaus zeigen tierexperimentelle Daten, dass dieses System auch das Herzmuskelzellwachstum beeinflusst und damit in engem Zusammenhang mit einer Herzhypertrophie steht $(3,6)$. Ein anderes Beispiel ist die Gefäßverkalkung, die ebenfalls über die Aktivierung des Vitamin-D-Rezeptors beeinflusst wird (1). Dies alles sind wichtige Befunde, denn „gerade die kardiovaskuläre Mortalität in Zusammenhang mit dem sekundären Hyperparathyreoidismus und der Niereninsuffizienz ist derzeit in unseren Fokus gerückt“, meinte Galle.

Mit Vitamin D sinken die Mortalitätsraten

Tatsächlich kann man mit einer Therapie mit Vitamin D mehr erreichen als die

1 Study to Evaluate Early Kidney disease

2 Dialysis Outcomes and Practice Patterns Study 
Kontrolle des Mineralhaushalts und des Knochenstoffwechsels. Sie verbessert das Überleben von Dialysepatienten, zeigte Ravi Thadhani, MD, MPH, Boston (USA), anhand einer großen retrospektiven Beobachtungsstudie aus dem letzten Jahr (11). Erhielten die Patienten aktiviertes Vitamin D (i.v), halbierte sich die Gesamtsterblichkeit und die kardiovaskuläre Mortalität der Patienten (28,6 versus 13,8 \% bzw. 14,8 versus 7,6 \%) - und zwar unabhängig von Alter, der ethnischen $\mathrm{Zu}$ gehörigkeit oder den Kalzium-, Phosphat- oder Parathormonspiegeln.

Auch die aktuellen Daten der DOPPS2Studie weisen der Supplementation mit Vitamin D einen Effekt bezüglich der Sterblichkeit zu. Insgesamt waren fast 45 \% der 17562 Dialysepatienten beider Studienkollektive zu irgendeinem Zeitpunkt während der Dialyse mit einem Vitamin-D-Analogon zur oralen oder intravenösen Applikation behandelt worden - eine Maßnahme, mit der die Gesamtsterblichkeit der Patienten signifikant um 11 \% reduziert werden konnte, wie die erste Risikoauswertung der Daten ergab, die bereits beim ASN-Jahreskongress 2005 präsentiert wurde.

Art des Vitamin-D-Analogons ist wichtig Doch welches Vitamin-D-Analogon eingesetzt worden war, war dabei nicht unerheblich: Im Vergleich zu einer Therapie mit oralem Vitamin D oder Calcitriol i.v. schnitt die Behandlung der Patienten mit Paricalcitol am besten ab. Nach der Adjustierung der Patientendaten hinsichtlich der demografischen Faktoren und der Begleiterkrankungen verringerte sich das Mortalitätsrisiko unter Paricalcitol hochsignifikant um 21\% ( $p<0,001$ ) (Abb. 1), unter einer i.v.-Gabe von Calcitriol dagegen nur um $10 \%$ ( $\mathrm{p}<$ $0,05)$. Hatten die Patienten orales Vitamin D eingenommen, war nur eine nichtsignifikante Reduktion der Gesamtsterblichkeit um 7 \% zu messen.

Auch nach einer weiteren Adjustierung der Daten hinsichtlich der Laborwerte war Paricalcitol die effektivste VitaminD-Therapie. Um 15 \% reduzierte sich das relative Sterberisiko unter Paricalcitol ( $p<0,05)$, während es unter Calcitriol i.v. nur um $9 \%(\mathrm{p}<0,05)$ und unter oralem Vitamin D nur um 5 \% (n.s.) sank. Vor kurzem wurden die Daten zusätzlich zwischen der nichtkardiovaskulären und der kardiovaskulären Mortalität differenziert, berichtete Prof. Jürgen
Bommer, Heidelberg, in seinem Ausblick auf die aktuelle zweite Risikoanalyse. Interessanterweise konnte mithilfe einer Vitamin-D-Therapie nur das nichtkardiovaskuläre Mortalitätsrisiko hochsignifikant gesenkt werden. Zwar reduzierte sich auch das kardiovaskuläre Risiko, wenn die Patienten Vitamin D einnahmen geringfügig, allerdings nicht signifikant. Mit Paricalcitol jedoch sank auch das kardiovaskuläre Risiko signifikant, berichtete Bommer.

Diese Hypothese stützen auch ältere Studienergebnisse. Zum Beispiel war Paricalcitol Calcitriol im Rahmen einer großen retrospektiven Kohortenstudie (10) bezüglich des Überlebens der Patienten signifikant überlegen. Lebten nach dreijähriger Therapie in der Paricalcitolgruppe noch 59\% der Patienten, waren dies im Calcitriolarm nur noch 51\% ( $p<0,001)$. Von Vorteil war auch ein Wechsel der Medikation auf Paricalcitol: 73\% der Patienten waren zwei Jahre nach der Umstellung der Therapie auf Paricalcitol noch am Leben $(\mathrm{n}=14862)$. Bei der Umstellung von Paricalcitol auf Calcitriol dagegen überlebten nur 64\% die beiden Folgejahre.

Die gute Nachricht ist, dass jetzt das Ergebnis verschiedener Studien, unter anderem auch in einer ganz aktuellen Longitudinalstudie (4), all diese Daten bestätigt. Hier war unter der Vitamin-DSupplementation mit Paricalcitol ebenfalls eine deutliche Reduktion der Mortalität zu sehen. „Damit haben wir übereinstimmende Studienergebnisse, die leider noch nicht zwingend eine Kausalität belegen - auch wenn ich dies glaube! Daher müssen weitere prospektive Studien folgen“, forderte Thadhani.

\section{Selektive Wirkung an der Nebenschild- drüse}

Dass vor allem Paricalcitol mit einer geringeren Mortalitätsrate einhergeht, ist für Thadhani keine Überraschung. Erhöhte Kalzium- und Phosphatspiegel sind mit einer höheren Sterblichkeitsrate bei Dialysepatienten assoziiert (2) so ältere Studiendaten. Paricalcitol dagegen erhöht diese Spiegel in geringerem Maße als andere Vitamin-D-Präparate, erklärte Thadhani. Daher kann man erwarten, dass es auch stärker die Mortalität senkt.

Der Grund dafür ist, dass unter Paricalcitol eine vergleichsweise geringe intestinale Kalzium- und Phosphataufnahme
Differenzielle Therapie nach K/DOQILeitlinien

Die aktuellen K/DOQI-Leitlinien (7) empfehlen unter anderem Paricalcitol, ein VitaminD-Analogon der dritten Generation, ausdrücklich zur differenziellen Therapie des 1,25-Vitamin-D-Mangels, wenn es darum geht, die PTH-Serumspiegel zu normalisieren ohne dabei gleichzeitig die Kalzium- und Phosphatserumspiegel zu erhöhen (8). Auch unter einer relativ hohen Dosierung von $\mathrm{Pa}$ ricalcitol bleiben diese Serumspiegel fast unverändert, weshalb die Substanz höher dosiert werden kann - und damit die Parathormonproduktion stärker reguliert - als zum Beispiel Calcitriol (9). Aber auch als Therapiealternative für Patienten, deren Kalzium- und Phosphatwerte über der in K/DOQI definierten Grenzwerte liegen, raten die Leitlinien zum Einsatz von Paricalcitol.

aus der Nahrung erfolgt (9), ergänzte Galle - ein Grund warum die aktuellen K/DOQI-Leitlinien Paricalcitol zur differenziellen Therapie des Vitamin-DMangels empfehlen (s. Kasten). Unerwünschte klinisch relevante Anstiege der Kalzium- und Phosphatserumspiegel werden somit - anders als zum Beispiel unter Calcitriol - deutlich reduziert.

Literatur

1 Andress DL. Kidney Int 2006; 69 (1): 33-43

2 Block GA et al. J Am Soc Nerphol 2004; 15 (8): 2208-2218

3 Cardus et al. Poster F-PO737, ASN 38th Renal Week Meeting, 2005

4 Kalantar-Zadeh K et al. Kidney Int 2006; 70 (4): 771-780

5 Kestenbaum B et al. J Am Soc Nephrol 2004; $16(2)$ : 520-528

$6 \quad$ Li QC et al. J Clin Invest 2002; 110 (2): 229238

7 National Kidney Foundation. Am J Kidney Dis 2003; 42 (suppl 3): S1-S201

8 Slatopolsky E et al. Kidney Int 2002; 62 (4): 1277-1284

9 Sprague SM et al. Kidney Int 2003; 6 (4): 1483-1490

10 Teng $M$ et al. N Engl J Med 2003; 349 (5): 446-456

11 Teng M et al. J Am Soc Nephrol 2005; 16 (4): 1115-1125

12 Xiang $W$ et al. Am J Physiol Endocrinol Metab 2005; 288 (1): E125-E132

13 Young EW et al. Poster TH-PO736, ASN 38 th Renal Week Meeting, 2005 